J. DIFFERENTIAL GEOMETRY

56 (2000) 111-132

\title{
$L_{p}$ AFFINE ISOPERIMETRIC INEQUALITIES
}

ERWIN LUTWAK, DEANE YANG \& GAOYONG ZHANG

\begin{abstract}
The $L_{p}$ analogues of the Petty projection inequality and the BusemannPetty centroid inequality are established.
\end{abstract}

An affine isoperimetric inequality compares two functionals associated with convex (or more general) bodies, where the ratio of the functionals is invariant under non-degenerate linear transformations. These isoperimetric inequalities are more powerful than their better-known Euclidean relatives.

This article deals with affine isoperimetric inequalities for centroid and projection bodies. Centroid bodies were attributed by Blaschke to Dupin (see e.g., the books of Schneider [32] and Leichtweiß [17] for references). If $K$ is an origin-symmetric convex body in Euclidean $n$ space, $\mathbb{R}^{n}$, then the centroid body of $K$ is the body whose boundary consists of the locus of the centroids of the halves of $K$ formed when $K$ is cut by codimension 1 subspaces. Blaschke (see Schneider [32] for references) conjectured that the ratio of the volume of a body to that of its centroid body attains its maximum precisely for ellipsoids. This conjecture was proven by Petty [27] who also extended the definition of centroid bodies and gave centroid bodies their name. When written as an inequality, Blaschke's conjecture is known as the Busemann-Petty centroid inequality. Busemann's name is attached to the inequality because Petty showed that Busemann's random simplex inequality ([5]) could be reinterpreted as what would become known as the BusemannPetty centroid inequality. In recent times, centroid bodies (and their

Received May 5, 2000. Research supported, in part, by NSF Grant DMS9803261. 
associated inequalities) have attracted increased attention (see e.g., Milman and Pajor [25, 26]). In retrospect, it can be seen that much if not all of this recent interest was inspired by Petty's seminal work [27].

Projection bodies are of newer vintage. They were introduced at the turn of the previous century by Minkowski. He showed that corresponding to each convex body $K$ in $\mathbb{R}^{n}$ is a unique origin-symmetric convex body $\Pi K$, the projection body of $K$, which can be defined (up to dilation) by the amazing fact that the following ratio is independent of the choice of 1-dimensional subspace $l$ of $\mathbb{R}^{n}$ : the length of the image of the orthogonal projection of $\Pi K$ onto $l$, to the $(n-1)$-dimensional volume of the image of the orthogonal projection of $K$ onto the codimension 1 subspace $l^{\perp}$. Interest in projection bodies was rekindled by three highly influential articles, which appeared in the latter half of the 60's, by Bolker [2], Petty [28], and Schneider [31]. Projection bodies have been the objects of intense investigation during the past three decades (see, e.g., Bourgain and Lindenstrauss [3], Schneider and Weil [33], Goodey and Weil [10], and the books of Schneider [32], Gardner [8], Leichtweiß [17], and Thompson [34]).

The fundamental inequality for projection bodies is the Petty projection inequality: Of all convex bodies of fixed (say, unit) volume, the ones whose polar projection bodies have maximal volume are precisely the ellipsoids. The inequality that states that simplices are precisely the bodies that minimize this volume is known as the Zhang projection inequality [35]. Petty [29] established the Petty projection inequality as a consequence of the Busemann-Petty centroid inequality. It was shown in [19] that this process could be reversed: the Busemann-Petty centroid inequality can be derived as a direct consequence of the Petty projection inequality. Both the Petty projection inequality and the BusemannPetty centroid inequality have come to be recognized as fundamental affine inequalities.

All centroid and projection bodies belong to the class $\mathcal{Z}^{n}$, of zonoids in $\mathbb{R}^{n}$. Zonoids can be defined as limits, with respect to the Hausdorff metric, of (Minkowski) sums of ellipsoids. The class $\mathcal{Z}^{n}$ arises naturally in various guises. For example, zonoids are the ranges of non-atomic $\mathbb{R}^{n}$-valued measures. They are also the polars of the unit balls of $n$ dimensional subspaces of $L_{1}([0,1])$.

To be more specific, this article concerns $L_{p}$-analogues of centroid and projection bodies. The $L_{p}$-analogues of centroid bodies have already appeared elsewhere. For example, the $L_{2}$-analogues of centroid bodies is an ellipsoid (called the Legendre ellipsoid) that appears in classical 
mechanics. However, the $L_{p}$-analogues of projection bodies are new. In order to correctly define them one needs the recently introduced (in [21] [22]) notion of $L_{p}$-curvature. Both the $L_{p}$-analogues of centroid bodies and the $L_{p^{-}}$-analogues of projection bodies belong to the class $\mathcal{Z}_{p}^{n}$ of $L_{p^{-}}$ zonoids. While less well known than the class of zonoids, the class $\mathcal{Z}_{p}^{n}$ is not new (see e.g., Schneider and Weil [33], and Goodey and Weil [10]). The bodies in $\mathcal{Z}_{p}^{n}$ are just the polars of the unit balls of $n$-dimensional subspaces of $L_{p}([0,1])$.

We shall derive the exact $L_{p}$-analogues of both the Busemann-Petty centroid inequality and the Petty projection inequality (as well as their equality conditions).

Let $S^{n-1}$ denote the unit sphere in Euclidean $n$-space, $\mathbb{R}^{n}$. Let $B$ denote the origin-centered standard unit ball in $\mathbb{R}^{n}$, and write $\omega_{n}$ for $V(B)$, the $n$-dimensional volume of $B$. Note that

$$
\omega_{n}=\pi^{n / 2} / \Gamma\left(1+\frac{n}{2}\right)
$$

defines $\omega_{n}$ for all non-negative real $n$ (not just the positive integers). For real $p \geq 1$, define $c_{n, p}$ by

$$
c_{n, p}=\frac{\omega_{n+p}}{\omega_{2} \omega_{n} \omega_{p-1}} .
$$

For each compact star-shaped about the origin $K \subset \mathbb{R}^{n}$, and each $p$ such that $1 \leq p \leq \infty$, let the norm $\|\cdot\|_{\Gamma_{p}^{*} K}$ on $\mathbb{R}^{n}$ be defined by

$$
\|x\|_{\Gamma_{p}^{*} K}=\left\{\frac{1}{c_{n, p} V(K)} \int_{K}|x \cdot y|^{p} d y\right\}^{1 / p}
$$

where $x \cdot y$ denotes the standard inner product of $x$ and $y$, and $V(K)$ denotes the volume of $K$. For the case $p=\infty$, this definition is to be interpreted as the limit as $p \rightarrow \infty$. The unit ball of the resulting $n$-dimensional $L_{p^{-}}$-space is denoted by $\Gamma_{p}^{*} K$, and called the polar $L_{p^{-}}$ centroid body of $K$. The (unusual) normalization above is chosen so that for the standard unit ball $B$ in $\mathbb{R}^{n}$, we have $\Gamma_{p}^{*} B=B$.

In [24] the following centro-affine inequality involving the volumes of $K$ and its polar $L_{p^{-}}$centroid body, $\Gamma_{p}^{*} K$, was established:

If $K$ is a star-shaped (about the origin) subset of $\mathbb{R}^{n}$, then for $1 \leq$ $p \leq \infty$,

$$
V(K) V\left(\Gamma_{p}^{*} K\right) \leq \omega_{n}^{2},
$$


with equality if and only if $K$ is an ellipsoid centered at the origin.

If $K$ is an origin-symmetric convex body, then $\Gamma_{\infty}^{*} K$ is just the polar, $K^{*}$, of $K$ where

$$
K^{*}=\left\{x \in \mathbb{R}^{n}: x \cdot y \leq 1, \quad \text { for all } y \in K\right\} .
$$

Thus, inequality $(*)$, for $p=\infty$, reduces to:

$$
V(K) V\left(K^{*}\right) \leq \omega_{n}^{2},
$$

with equality if and only if $K$ is an ellipsoid.

This is the well known Blaschke-Santaló inequality.

In light of the Blaschke-Santaló inequality, a stronger inequality than $(*)$ was conjectured in [24]. This stronger inequality is the inequality of our first theorem:

Theorem 1. If $K$ is a star body (about the origin) in $\mathbb{R}^{n}$, then for $1 \leq p<\infty$,

$$
V\left(\Gamma_{p} K\right) \geq V(K)
$$

with equality if and only if $K$ is an ellipsoid centered at the origin.

Here $\Gamma_{p} K$, the $L_{p}$-centroid body of $K$, is just the polar of $\Gamma_{p}^{*} K$.

For the case $p=1$ the inequality of Theorem 1 is known as the Busemann-Petty centroid inequality [27] (see also the books of Schneider [32], Gardner [8], and Leichtweiß [17]). The case $p=2$ is also well known and goes back to at least, to Blaschke [1] (see also Lindenstrauss and Milman [18], Milman and Pajor [25] [26], Petty [27], and also [23]). For all other values of $p$ the inequality of Theorem 1 is new.

The inequality closely related to the Busemann-Petty centroid inequality is known as the Petty projection inequality [29] (see also the books of Schneider [32], Gardner [8], and Leichtweiß [17]). The $L_{p^{-}}$ version of the Petty projection inequality will also be established in this article.

It will be convenient throughout to restrict our attention to only those convex (and star-shaped) bodies which contain the origin in their interiors. This assumption will tacitly be made throughout.

\section{The $L_{p}$ version of the Petty Projection Inequality}

If $K$ is a convex body (i.e., a compact, convex subset containing the origin in its interior) in $\mathbb{R}^{n}$, then its support function, $h_{K}=h(K, \cdot)$ : $\mathbb{R}^{n} \rightarrow[0, \infty)$, can be defined for $x \in \mathbb{R}^{n}$ by $h(K, x)=\max \{x \cdot y: y \in K\}$. 
For $p \geq 1$, convex bodies $K, L$, and $\varepsilon>0$, the Minkowski-Firey $L_{p}$-combination $K+\varepsilon \cdot L$ is defined as the convex body whose support function is given by

$$
h(K+\varepsilon \cdot L, \cdot)^{p}=h(K, \cdot)^{p}+\varepsilon h(L, \cdot)^{p} .
$$

Although Minkowski-Firey addition and scalar multiplication depend on $p$, our notation does not reflect this fact. Minkowski-Firey combinations of convex bodies were defined and studied by Firey [6] (who called them $p$-means of convex bodies).

For $p \geq 1$, the $L_{p}$-mixed volume, $V_{p}(K, L)$, of the convex bodies $K, L$ was defined in [21] by:

$$
\frac{n}{p} V_{p}(K, L)=\lim _{\varepsilon \rightarrow 0^{+}} \frac{V(K+\varepsilon \cdot L)-V(K)}{\varepsilon} .
$$

That this limit exists was demonstrated in [21].

It was shown in [21], that corresponding to each convex body, $K$, that contains the origin in its interior, there is a positive Borel measure, $S_{p}(K, \cdot)$, on $S^{n-1}$ such that

$$
V_{p}(K, Q)=\frac{1}{n} \int_{S^{n-1}} h(Q, u)^{p} d S_{p}(K, u)
$$

for each convex body $Q$. The measure $S_{1}(K, \cdot)$ is just the classical surface area measure of $K$. This measure is usually denoted by $S(K, \cdot)$ or $S_{K}$.

For positive real $p$, let $\mathrm{C}_{p}$ denote the spherical $L_{p}$-cosine transform on $S^{n-1}$; i.e., for each positive Borel measure, $\mu$, on $S^{n-1}$, let $\mathrm{C}_{p} \mu$ be the continuous function on $S^{n-1}$ defined by

$$
\left(\mathrm{C}_{p} \mu\right)(u)=\left\{\frac{1}{n \omega_{n} c_{n-2, p}} \int_{S^{n-1}}|u \cdot v|^{p} d \mu(v)\right\}^{1 / p},
$$

for each $u \in S^{n-1}$. The unusual normalization above was chosen so that for Lebesgue measure, $S$, on $S^{n-1}$, we have $\mathrm{C}_{p} S=1$.

For $p=1$ the spherical $L_{p}$-cosine transform is just the well-known spherical cosine transform which is closely related to the spherical Radon transform (see e.g., Goodey and Weil [9], [10]). The operator $\mathrm{C}_{1}$ will be written simply as C. For $p>1$ the spherical $L_{p}$-cosine transform is also well known (see e.g., Koldobsky [11], [12], [13], [14], [15], [16], and Rubin [30] for applications and references). 
For each convex body $K$, define the $L_{p}$-projection body, $\Pi_{p} K$, of $K$ to be the origin-symmetric convex body whose support function is given by

$$
h\left(\Pi_{p} K, \cdot\right)=\mathrm{C}_{p} S_{p}(K, \cdot) .
$$

The unusual normalization above is chosen so that for the unit ball, $B$, we have $\Pi_{p} B=B$.

Just as $\Gamma_{p}^{*} K$, rather than $\left(\Gamma_{p} K\right)^{*}$, is used to denote the polar body of $\Gamma_{p} K$, we will denote the polar of the body $\Pi_{p} K$ by $\Pi_{p}^{*} K$, rather than $\left(\Pi_{p} K\right)^{*}$.

A $p=1$ is often suppressed. The convex body $\Pi K$ is known simply as the projection body of $K$. Note again that we have adopted a normalization that differs from the classical in that $\Pi B$ is simply $B$ (rather than the classical $\omega_{n-1} B$ ). We note again that in order to define the $L_{p}$-projection body of a convex body, for $p>1$, the notion of an $L_{p}$-curvature measure (or function) is critical.

One of the classical affine isoperimetric inequalities is the Petty projection inequality [29]. It states that for each convex body $K$ in $\mathbb{R}^{n}$,

$$
V(K)^{n-1} V\left(\Pi^{*} K\right) \leq \omega_{n}^{n},
$$

with equality if and only if $K$ is an ellipsoid.

The Petty projection inequality is the statement that the quantity $V(K)^{n-1} V\left(\Pi^{*} K\right)$ is maximized precisely when the body $K$ is an ellipsoid. The Zhang projection inequality [35] states that this quantity is minimized precisely by simplices.

We will establish the $L_{p}$-analogue of the Petty projection inequality:

Theorem 2. If $K$ is a convex body in $\mathbb{R}^{n}$, then for $1<p<\infty$,

$$
V(K)^{(n-p) / p} V\left(\Pi_{p}^{*} K\right) \leq \omega_{n}^{n / p},
$$

with equality if and only if $K$ is an ellipsoid centered at the origin.

The special case $p=2$ of Theorem 2 , and its reverse inequality, can be found in [23].

\section{Outline of proof}

For real $p \geq 1$ define the $L_{p}$-Petty projection product of a convex body $K$ by

$$
\mathrm{p}_{p}(K)=\omega_{n}^{-n / p} V(K)^{(n-p) / p} V\left(\Pi_{p}^{*} K\right) .
$$


For real $p \geq 1$ define the $L_{p}$-Busemann-Petty ratio of the star body $K$ by

$$
\mathrm{b}_{p}(K)=V(K) / V\left(\Gamma_{p} K\right) .
$$

Note that while $\mathrm{p}_{p}$ is defined only for convex bodies, $\mathrm{b}_{p}$ is defined for all star bodies.

From the definition of $L_{p}$-projection body, it follows immediately that for $\lambda>0$ we have $\Pi_{p} \lambda K=\lambda^{(n-p) / p} \Pi_{p} K$, where $\lambda K=\{\lambda x: x \in$ $K\}$ is the dilate of $K$ by a factor of $\lambda$. Thus $\Pi_{p}^{*} \lambda K=\lambda^{(p-n) / p} \Pi_{p}^{*} K$ which shows that the functional $\mathrm{p}_{p}$ is dilation invariant. In the next section we prove (Lemma 2 ) that $\mathrm{p}_{p}$ is in fact a $\mathrm{GL}(n)$-invariant functional: For each convex body $K$,

$$
\mathrm{p}_{p}(\phi K)=\mathrm{p}_{p}(K), \quad \text { for all } \phi \in \mathrm{GL}(n),
$$

where $\phi K=\{\phi x: x \in K\}$ is the image of $K$ under $\phi$.

In order to demonstrate the existence of a convex body at which $\mathrm{p}_{p}$ attains a maximum, proceed as follows: Let $\hat{\mathrm{p}}_{p}$ denote the supremum of the functional $\mathrm{p}_{p}$ taken over all convex bodies. Let $K_{i}$ denote a maximizing sequence for $\mathrm{p}_{p}$; i.e., $K_{i}$ is a sequence of convex bodies such that

$$
\lim _{i \rightarrow \infty} \mathrm{p}_{p}\left(K_{i}\right)=\hat{\mathrm{p}}_{p}
$$

In the next section we will use a class reduction technique to show (Lemma 7 ) that, unless the body $K_{i}$ is origin-symmetric, there exists an origin-symmetric body $\bar{K}_{i}$ such that $\mathrm{p}_{p}\left(K_{i}\right)<\mathrm{p}_{p}\left(\bar{K}_{i}\right)$. Thus it may be assumed that the original maximizing sequence consists solely of bodies that are origin-symmetric. A classical theorem of John (see e.g., Thompson [34]) yields the existence of a sequence of origin-symmetric ellipsoids $E_{i}$ such that $E_{i} \subset K_{i} \subset \sqrt{n} E_{i}$. But since $\mathrm{p}_{p}$ is a $\operatorname{GL}(n)$ invariant functional, we may assume that the maximizing sequence $K_{i}$ is such that, for all $i$

$$
B \subset K_{i} \subset \sqrt{n} B,
$$

where $B$ denotes the origin-centered unit ball. The Blaschke selection theorem now guarantees the existence of a body at which $\mathrm{p}_{p}$ attains a maximum. Since this maximizing body is the limit (with respect to the Hausdorff metric) of a subsequence of the $K_{i}$, it follows that this maximizing body contains the origin (in fact the interior of the unit ball) in its interior.

We will use a class reduction technique to show that all bodies at which the maximum of $\mathrm{p}_{p}$ is attained must be sufficiently smooth. This 
reduction will be critical in our proof. The proof is completed by the fundamental fact (Lemma 14) that Steiner symmetrization intertwines with the polar $L_{p}$-projection operator.

Finally, to prove that $b_{p}<1$ for all star bodies (except ellipsoids), we will use a class reduction result to show that this follows from the fact that $\mathrm{p}_{p}<1$ for a small class of convex bodies.

Although we will not use either the Petty projection inequality nor the Busemann-Petty centroid inequality to prove their $L_{p}$ analogues, we do not wish to reprove these classical inequalities. Thus, throughout we shall restrict our attention solely to the case of real $p>1$. Of course, by taking limits (as $p \rightarrow 1$ ) one may recover the classical inequalities (but not necessarily their equality conditions) from their $L_{p}$ analogues. We note again that we will be tacitly assuming throughout that all bodies contain the origin in their interiors.

\section{Mixed and dual mixed volumes and the operators $\Pi_{p}^{*}$ and $\Gamma_{p}$}

For quick reference, we recall some basic properties of $L_{p}$-mixed and dual mixed volumes. Some recent applications of dual mixed volumes can be found in [7], [36], [37] and [38]. For general reference the reader may wish to consult the books of Gardner [8] and Schneider [32]. We emphasize again that we are assuming throughout that $1<p<\infty$ and that our convex (and star-shaped) bodies all contain the origin in their interiors.

The radial function, $\rho_{K}=\rho(K, \cdot): \mathbb{R}^{n} \backslash\{0\} \rightarrow[0, \infty)$, of a compact, star-shaped (about the origin) $K \subset \mathbb{R}^{n}$, is defined, for $x \neq 0$, by $\rho(K, x)=\max \{\lambda \geq 0: \lambda x \in K\}$. If $\rho_{K}$ is positive and continuous, call $K$ a star body (about the origin). Two star bodies $K$ and $L$ are said to be dilates (of one another) if $\rho_{K}(u) / \rho_{L}(u)$ is independent of $u \in S^{n-1}$.

If $K$ is a convex body, then it follows from the definitions of support and radial functions, and the definition of polar body, that

$$
h_{K^{*}}=1 / \rho_{K} \quad \text { and } \quad \rho_{K^{*}}=1 / h_{K} .
$$

For star bodies $K, L$, and $\varepsilon>0$, the $L_{p}$-harmonic radial combination $K \tilde{+} \varepsilon \cdot L$ is the star body defined by

$$
\rho(K \widetilde{+} \varepsilon \cdot L, \cdot)^{-p}=\rho(K, \cdot)^{-p}+\varepsilon \rho(L, \cdot)^{-p} .
$$


While this addition and scalar multiplication are obviously dependent on $p$, we have not made this explicit in our notation. The dual mixed volume $\widetilde{V}_{-p}(K, L)$ of the star bodies $K, L$, can be defined by

$$
\frac{n}{-p} \widetilde{V}_{-p}(K, L)=\lim _{\varepsilon \rightarrow 0^{+}} \frac{V(K \widetilde{+} \varepsilon \cdot L)-V(K)}{\varepsilon} .
$$

The definition above and the polar coordinate formula for volume give the following integral representation of the dual mixed volume $\widetilde{V}_{-p}(K, L)$ of the star bodies $K, L$ :

$$
\widetilde{V}_{-p}(K, L)=\frac{1}{n} \int_{S^{n-1}} \rho_{K}^{n+p}(v) \rho_{L}^{-p}(v) d S(v),
$$

where the integration is with respect to spherical Lebesgue measure $S$ on $S^{n-1}$.

From the definition of support function, it follows immediately that for a convex body $K$, an $x \in \mathbb{R}^{n}$, and a $\phi \in \mathrm{SL}(n)$, we have $h_{\phi K}(x)=$ $h_{K}\left(\phi^{t} x\right)$, where $\phi^{t}$ denotes the transpose of $\phi$ and $\phi K=\{\phi x: x \in K\}$ is the image of $K$ under $\phi$. This and the definition of a MinkowskiFirey $L_{p^{-}}$-combination show that for a Minkowski-Firey $L_{p^{-}}$-combination of convex bodies $K$ and $L$, we have

$$
\phi(K+\varepsilon \cdot L)=\phi K+\varepsilon \cdot \phi L .
$$

This observation together with the definition of the $L_{p}$-mixed volume $V_{p}$ shows that for $\phi \in \mathrm{SL}(n)$ and convex bodies $K, L$ we have $V_{p}(\phi K, \phi L)=$ $V_{p}(K, L)$ or equivalently

$$
V_{p}(\phi K, L)=V_{p}\left(K, \phi^{-1} L\right) .
$$

From the definition of radial function, it follows immediately that for a star body $K$, an $x \in \mathbb{R}^{n}$, and a $\phi \in \mathrm{SL}(n)$, we have $\rho_{\phi K}(x)=$ $\rho_{K}\left(\phi^{-1} x\right)$. This and the definition of an $L_{p}$-harmonic radial combination shows that for an $L_{p}$-harmonic radial combination of star bodies $K$ and $L$,

$$
\phi(K \widetilde{+} \varepsilon \cdot L)=\phi K \widetilde{+} \varepsilon \cdot \phi L .
$$

This observation together with the definition of the dual mixed volume $\widetilde{V}_{-p}$ shows that for $\phi \in \mathrm{SL}(n)$ and star bodies $K, L$ we have $\widetilde{V}_{-p}(\phi K, \phi L)=\widetilde{V}_{-p}(K, L)$ or equivalently

$$
\widetilde{V}_{-p}(\phi K, L)=\widetilde{V}_{-p}\left(K, \phi^{-1} L\right) .
$$


We shall require two basic inequalities regarding the $L_{p}$-mixed volumes $V_{p}$ and the dual mixed volumes $\widetilde{V}_{-p}$. The $L_{p}$ analogue of the classical Minkowski inequality states that for convex bodies $K, L$,

$$
V_{p}(K, L) \geq V(K)^{(n-p) / n} V(L)^{p / n},
$$

with equality if and only if $K$ and $L$ are dilates. The $L_{p}$-Minkowski inequality was established in [21] by using the Minkowski inequality. The basic inequality for the dual mixed volumes $\widetilde{V}_{-p}$ is that for star bodies $K, L$,

$$
\widetilde{V}_{-p}(K, L) \geq V(K)^{(n+p) / n} V(L)^{-p / n},
$$

with equality if and only if $K$ and $L$ are dilates. This inequality is an immediate consequence of the Hölder inequality and the integral representation (6).

From the definition of the $L_{p}$-mixed volume $V_{p}$ it follows immediately that for each convex body $K$,

$$
V_{p}(K, K)=V(K)
$$

From the definition of the dual mixed volumes $\widetilde{V}_{-p}$ it follows immediately that for each star body $K$,

$$
\widetilde{V}_{-p}(K, K)=V(K) .
$$

Note that (11) holds only for convex bodies, while identity (12) holds for all star bodies.

An immediate consequence of the dual mixed volume inequality (10) and identity (12) is that if for star bodies $K, L$ we have

$$
\widetilde{V}_{-p}(Q, K) / V(Q)=\widetilde{V}_{-p}(Q, L) / V(Q),
$$

for all star bodies $Q$ which belong to some class that contains both $K$ and $L$, then in fact $K=L$.

Lemma 1. If $K$ is a star body and $L$ is a convex body in $\mathbb{R}^{n}$, then

$$
V_{p}\left(L, \Gamma_{p} K\right)=\frac{\omega_{n}}{V(K)} \widetilde{V}_{-p}\left(K, \Pi_{p}^{*} L\right)
$$

Proof. From the definition of the $L_{p}$-centroid body of $K$,

$$
h_{\Gamma_{p} K}^{p}(u)=\frac{1}{c_{n, p} V(K)} \int_{K}|u \cdot x|^{p} d x
$$


the integral representation (3), Fubini's theorem, (5), and the integral representation (6), it follows that

$$
\begin{aligned}
V_{p}\left(L, \Gamma_{p} K\right) & =\frac{1}{n} \int_{S^{n-1}} h_{\Gamma_{p} K}^{p}(u) d S_{p}(L, u) \\
& =\frac{1}{n} \int_{S^{n-1}}\left(\frac{1}{c_{n, p} V(K)} \int_{K}|u \cdot x|^{p} d x\right) d S_{p}(L, u) \\
& =\frac{1}{n(n+p) c_{n, p} V(K)} \int_{S^{n-1}} \int_{S^{n-1}}|u \cdot v|^{p} \rho_{K}^{n+p}(v) d S(v) d S_{p}(L, u) \\
& =\frac{\omega_{n}}{n V(K)} \int_{S^{n-1}} \rho_{K}^{n+p}(v) h_{\Pi_{p} L}^{p}(v) d S(v) \\
& =\frac{\omega_{n}}{V(K)} \widetilde{V}_{-p}\left(K, \Pi_{p}^{*} L\right) \text {. q.e.d. }
\end{aligned}
$$

For $p=1$ the identity of Lemma 1 was presented in [20].

From (5) and the transformation rules for support and radial functions we see that for a convex body $K$ and $\phi \in \mathrm{SL}(n)$

$$
(\phi K)^{*}=\phi^{-t} K
$$

where $\phi^{-t}$ denotes the inverse of the transpose of $\phi$.

An immediate consequence of the definition of the $L_{p}$-centroid body of $K$ is that for $\phi \in \mathrm{SL}(n)$,

$$
\Gamma_{p} \phi K=\phi \Gamma_{p} K .
$$

Lemma 2. If $K$ is a convex body in $\mathbb{R}^{n}$ that contains the origin in its interior, $1<p<\infty$, and $\phi \in \mathrm{SL}(n)$, then

$$
\Pi_{p} \phi K=\phi^{-t} \Pi_{p} K .
$$

Proof. From Lemma 1, followed by (7), (14), Lemma 1 again, and (8) we have for each star body $Q$,

$$
\begin{aligned}
\omega_{n} \widetilde{V}_{-p}\left(Q, \Pi_{p}^{*} \phi K\right) / V(Q) & =V_{p}\left(\phi K, \Gamma_{p} Q\right) \\
& =V_{p}\left(K, \phi^{-1} \Gamma_{p} Q\right) \\
& =V_{p}\left(K, \Gamma_{p} \phi^{-1} Q\right) \\
& =\omega_{n} \widetilde{V}_{-p}\left(\phi^{-1} Q, \Pi_{p}^{*} K\right) / V(Q) \\
& =\omega_{n} \widetilde{V}_{-p}\left(Q, \phi \Pi_{p}^{*} K\right) / V(Q) .
\end{aligned}
$$


But $\widetilde{V}_{-p}\left(Q, \Pi_{p}^{*} \phi K\right) / V(Q)=\widetilde{V}_{-p}\left(Q, \phi \Pi_{p}^{*} K\right) / V(Q)$ for all star bodies $Q$ implies that

$$
\Pi_{p}^{*} \phi K=\phi \Pi_{p}^{*} K
$$

and now (13) yields the desired conclusion. q.e.d.

Lemma 2 for $p=1$ was established by Petty [28] by using a completely different argument.

In Lemma 1, take $L=\Gamma_{p} K$, use (11) and get:

Lemma 3. If $K$ is a star body in $\mathbb{R}^{n}$, then

$$
V\left(\Gamma_{p} K\right)=\omega_{n} \widetilde{V}_{-p}\left(K, \Pi_{p}^{*} \Gamma_{p} K\right) / V(K) .
$$

In Lemma 1, take $K=\Pi_{p}^{*} L$, use (12) and get:

Lemma 4. If $L$ is a convex body in $\mathbb{R}^{n}$, then

$$
V_{p}\left(L, \Gamma_{p} \Pi_{p}^{*} L\right)=\omega_{n}
$$

For $p=1$ this identity was obtained in [20].

Recall that the $L_{p}$-Petty projection product of the convex body $K$ was defined by

$$
\mathrm{p}_{p}(K)=V(K)^{(n-p) / p} V\left(\Pi_{p}^{*} K\right) \omega_{n}^{-n / p}
$$

while the $L_{p}$-Busemann-Petty ratio of the star body $K$ was defined by

$$
\mathrm{b}_{p}(K)=V(K) / V\left(\Gamma_{p} K\right) .
$$

Our ultimate goal is to show that both $\mathrm{p}_{p}$ and $\mathrm{b}_{p}$ never exceed 1 and that in fact they will attain the value of 1 only on ellipsoids.

From Lemma 3 and the dual mixed volume inequality (10) we immediately obtain:

Lemma 5. If $K$ is a star body in $\mathbb{R}^{n}$ and $1<p<\infty$, then

$$
\mathrm{p}_{p}\left(\Gamma_{p} K\right) \geq \mathrm{b}_{p}(K)
$$

with equality if and only if $K$ and $\Pi_{p}^{*} \Gamma_{p} K$ are dilates. 
From Lemma 4 and the $L_{p}$ mixed volume inequality (9) we immediately obtain:

Lemma 6. If $K$ is a convex body in $\mathbb{R}^{n}$ and $1<p<\infty$, then

$$
\mathrm{b}_{p}\left(\Pi_{p}^{*} K\right) \geq \mathrm{p}_{p}(K)
$$

with equality if and only if $K$ and $\Gamma_{p} \Pi_{p}^{*} K$ are dilates.

Combine Lemmas 5 and 6 to get:

Lemma 7 . If $K$ is a convex body in $\mathbb{R}^{n}$ then

$$
\mathrm{p}_{p}\left(\Gamma_{p} \Pi_{p}^{*} K\right) \geq \mathrm{p}_{p}(K)
$$

with equality if and only if $K$ and $\Gamma_{p} \Pi_{p}^{*} K$ are dilates.

Throughout, a convex body will be called smooth if its boundary is $C^{2}$ with everywhere positive curvature. Thus smooth bodies have curvature bounded away from 0 and $\infty$. In the literature smooth bodies are often called $C_{+}^{2}$.

Petty [27] proved that all centroid bodies are smooth. The fact that this is also the case for the $L_{p}$ analogues of centroid bodies for $p>1$ is much easier to see. Lemma 7 shows that any body at which $\mathrm{p}_{p}$ attains a maximum must be smooth and origin-symmetric. Such class reduction methods were presented in [19].

Our aim is to show that given any maximal body $K$ for $\mathrm{p}_{p}$ and any direction $u \in S^{n-1}$, the midpoints of the chords of $K$ in the direction $u$ are coplanar. This together with the classical Bertrand-Brunn theorem (see e.g., Thompson [34]) will allow us to conclude that $K$ is an ellipsoid. To this end a few preliminary lemmas are needed.

\section{Some basic facts and lemmas}

First we shall need the following trivial elementary inequality:

Lemma 8. If $a, b \geq 0$ and $c, d>0$, then for $p>1$

$$
(a+b)^{p}(c+d)^{1-p} \leq a^{p} c^{1-p}+b^{p} d^{1-p},
$$

with equality if and only if $a d=b c$.

Rewriting the inequality as

$$
\lambda \frac{a}{c}+(1-\lambda) \frac{b}{d} \leq\left\{\lambda\left(\frac{a}{c}\right)^{p}+(1-\lambda)\left(\frac{b}{d}\right)^{p}\right\}^{1 / p},
$$


with $\lambda=c /(c+d)$, shows that this is a direct consequence of the convexity of the function $t \mapsto t^{p}$.

If $K$ is a convex body and $\xi$ is a subspace of codimension 1, then $\mathrm{S}_{\xi} K$ will denote the Steiner symmetral of $K$ with respect to $\xi$. Thus if $K \subset \mathbb{R}^{n-1} \times \mathbb{R}$, then

$$
\mathrm{S}_{\mathbb{R}^{n-1}} K=\left\{\left(x, \frac{1}{2} t+\frac{1}{2} s\right) \in \mathbb{R}^{n-1} \times \mathbb{R}:(x, t) \in K,(x,-s) \in K\right\} .
$$

If $K \subset \mathbb{R}^{n-1} \times \mathbb{R}$ is a convex body given by

$$
K=\left\{(x, t) \in \mathbb{R}^{n-1} \times \mathbb{R}:-g(x) \leq t \leq f(x), x \in K_{o}\right\},
$$

where $K_{o}$ is the image of the orthogonal projection of $K$ onto $\mathbb{R}^{n-1}$ and $f, g: K_{o} \rightarrow \mathbb{R}$, then

$$
\begin{aligned}
& \mathrm{S}_{\mathbb{R}^{n-1}} K= \\
& \quad\left\{(x, t) \in \mathbb{R}^{n-1} \times \mathbb{R}:-\frac{1}{2}(f(x)+g(x)) \leq t \leq \frac{1}{2}(f(x)+g(x)), x \in K_{o}\right\},
\end{aligned}
$$

We will need the following often used fact:

Lemma 9. Suppose $K, L \subset \mathbb{R}^{n-1} \times \mathbb{R}$ are convex bodies. Then

$$
\mathrm{S}_{\mathbb{R}^{n-1}} K^{*} \subset L^{*}
$$

if and only if

$$
h_{K}(x, t)=1=h_{K}(x,-s), \text { with } t \neq-s \Longrightarrow h_{L}\left(x, \frac{1}{2} t+\frac{1}{2} s\right) \leq 1 .
$$

In addition if $\mathrm{S}_{\mathbb{R}^{n-1}} K^{*}=L^{*}$, then $h_{K}(x, t)=1=h_{K}(x,-s)$, with $t \neq-s$ must imply $h_{L}\left(x, \frac{1}{2} t+\frac{1}{2} s\right)=1$.

Lemma 9 is an immediate consequence of the definition of Steiner symmetrization, identities (5) and the obvious fact that for each body $Q$, we have $x \in Q \backslash \partial Q$ if and only if $\rho_{Q}(x)>1$.

Lemma 10. Suppose $K \subset \mathbb{R}^{n-1} \times \mathbb{R}$ is a smooth convex body given by

$$
K=\left\{(x, t) \in \mathbb{R}^{n-1} \times \mathbb{R}:-g(x) \leq t \leq f(x), x \in K_{o}\right\},
$$

where $K_{o}$ is the image of the orthogonal projection of $K$ onto $\mathbb{R}^{n-1}$ and $f, g: K_{o} \rightarrow \mathbb{R}$. If $h: S^{n-1} \rightarrow \mathbb{R}$ is a continuous function then

$$
\begin{aligned}
\int_{S^{n-1}} & \frac{h(u)}{\kappa_{K}(u)} d S(u) \\
= & \int_{\operatorname{int} K_{o}}\left[h\left(u_{x}^{+}\right) \sqrt{1+|\nabla f(x)|^{2}}+h\left(u_{x}^{-}\right) \sqrt{1+|\nabla g(x)|^{2}}\right] d x
\end{aligned}
$$


where $\kappa_{K}(u)$ is the Gauss curvature of $\partial K$ at the point of $\partial K$ whose outer unit normal is $u$, while $u_{x}^{+}$is the outer unit normal to $K$ at $(x, f(x))$ and $u_{x}^{-}$is the outer unit normal to $K$ at $(x,-g(x))$.

The Lemma follows from the formula for the surface area of a graph and the fact that the Gauss curvature is equal to the Jacobian of the Gauss map.

Suppose $A$ is the interior of a convex subset of $\mathbb{R}^{n-1}$ and $f: A \rightarrow \mathbb{R}$ is a $C^{1}$ function, then $\langle f\rangle: A \rightarrow \mathbb{R}$ is the function defined by

$$
\langle f\rangle(x)=f(x)-x \cdot \nabla f(x),
$$

for each $x \in A$. Note that $\langle\cdot\rangle$ is a linear operator; i.e., if $f_{1}, f_{2}: A \rightarrow \mathbb{R}$ and $\lambda_{1}, \lambda_{2} \in \mathbb{R}$, then

$$
\left\langle\lambda_{1} f_{1}+\lambda_{2} f_{2}\right\rangle=\lambda_{1}\left\langle f_{1}\right\rangle+\lambda_{2}\left\langle f_{2}\right\rangle
$$

We shall need the fact that the kernel of the operator $\langle\cdot\rangle$ consists only of linear functions; i.e.,

$$
\langle f\rangle(x)=0 \quad \text { for all } x \in A \quad \Longrightarrow \quad f \text { is linear on } A .
$$

Finally, we shall require the trivial observation that if $A$ is originsymmetric, then

$$
\begin{aligned}
f_{1}(-x)=f_{2}(x) \quad & \text { for all } x \in A \Longrightarrow \\
& \left\langle f_{1}\right\rangle(-x)=\left\langle f_{2}\right\rangle(x) \text { for all } x \in A .
\end{aligned}
$$

Lemma 11. Suppose $K \subset \mathbb{R}^{n-1} \times \mathbb{R}$ is a smooth convex body given by

$$
K=\left\{(x, t) \in \mathbb{R}^{n-1} \times \mathbb{R}:-g(x) \leq t \leq f(x), x \in K_{o}\right\},
$$

where $K_{o}$ is the image of the orthogonal projection of $K$ onto $\mathbb{R}^{n-1}$ and $f, g: K_{o} \rightarrow \mathbb{R}$. Then

$$
h_{K}(-\nabla f(x), 1)=\langle f\rangle(x)
$$

and

$$
h_{K}(-\nabla g(x),-1)=\langle g\rangle(x)
$$

for all $x \in \operatorname{int} K_{o}$. 
To see this note that for $x \in \operatorname{int} K_{o}$, the outer unit normal to $\partial K$ at the point $(x, f(x))$ is

$$
u_{x}^{+}=\frac{(-\nabla f(x), 1)}{\sqrt{1+|\nabla f(x)|^{2}}}
$$

and the outer unit normal to $\partial K$ at the point $(x,-g(x))$ is

$$
u_{x}^{-}=\frac{(-\nabla g(x),-1)}{\sqrt{1+|\nabla g(x)|^{2}}} \text {. }
$$

Hence

$$
h_{K}\left(\frac{(-\nabla f(x), 1)}{\sqrt{1+|\nabla f(x)|^{2}}}\right)=h_{K}\left(u_{x}^{+}\right)=(x, f(x)) \cdot u_{x}^{+}=\frac{f(x)-x \cdot \nabla f(x)}{\sqrt{1+|\nabla f(x)|^{2}}}
$$

and

$$
h_{K}\left(\frac{(-\nabla g(x),-1)}{\sqrt{1+|\nabla g(x)|^{2}}}\right)=h_{K}\left(u_{x}^{-}\right)=(x,-g(x)) \cdot u_{x}^{-}=\frac{g(x)-x \cdot \nabla g(x)}{\sqrt{1+|\nabla g(x)|^{2}}} .
$$

The homogeneity (of degree 1 ) of $h_{K}$ now gives the identities of the Lemma.

As an aside, we note that since $K$ contains the origin in its interior, it follows that for $x \in \operatorname{int} K_{o}$

$$
\langle f\rangle(x)=h_{K}(-\nabla f(x), 1)>0,
$$

and

$$
\langle g\rangle(x)=h_{K}(-\nabla g(x),-1)>0 .
$$

\section{Steiner symmetrization and the operator $\Pi_{p}^{*}$}

Lemma 12. Suppose $K \subset \mathbb{R}^{n-1} \times \mathbb{R}$ is a smooth convex body given by

$$
K=\left\{(x, t) \in \mathbb{R}^{n-1} \times \mathbb{R}:-g(x) \leq t \leq f(x), x \in K_{o}\right\},
$$

where $K_{o}$ is the image of the orthogonal projection of $K$ onto $\mathbb{R}^{n-1}$ and $f, g: K_{o} \rightarrow \mathbb{R}$. Then the support function of $\Pi_{p} K$ at $(y, t) \in \mathbb{R}^{n-1} \times \mathbb{R}$ is given by

$$
\begin{aligned}
& n \omega_{n} c_{n-2, p} h_{\Pi_{p} K}^{p}(y, t)= \\
& \quad \int_{\operatorname{int} K_{o}}\left[|t-y \cdot \nabla f(x)|^{p}\langle f\rangle(x)^{1-p}+|t+y \cdot \nabla g(x)|^{p}\langle g\rangle(x)^{1-p}\right] d x .
\end{aligned}
$$


Proof. It was shown in [21] that the $L_{p}$-surface area measure $S_{p}(K, \cdot)$ is absolutely continuous with respect to the classical surface area measure $S_{K}$ and that the Radon-Nikodym derivative

$$
\frac{d S_{p}(K, \cdot)}{d S_{K}}=h_{K}^{1-p}
$$

Since $K$ is smooth, the measure $S_{K}$ is absolutely continuous with respect to spherical Lebesgue measure $S$ and the Radon-Nikodym derivative

$$
\frac{d S_{K}}{d S}=1 / \kappa_{K}
$$

where $\kappa_{K}: S^{n-1} \rightarrow(0, \infty)$ is the Gauss curvature of $\partial K$ viewed as a function of the outer normals (i.e., $\kappa_{K}(u)$, for $u \in S^{n-1}$, is the Gauss curvature at the point of $\partial K$ whose outer unit normal is $u$ ).

These observations together with the definition of $\Pi_{p} K$ show that for $(y, t) \in \mathbb{R}^{n-1} \times \mathbb{R}$

$$
h_{\Pi_{p} K}^{p}(y, t)=\frac{1}{n \omega_{n} c_{n-2, p}} \int_{S^{n-1}}|(y, t) \cdot u|^{p} \frac{h_{K}^{1-p}(u)}{\kappa_{K}(u)} d S(u) .
$$

Now if $h: \mathbb{R}^{n} \rightarrow \mathbb{R}$ is any continuous function that is homogeneous of degree 1 , then from $\left(16^{+}\right),\left(16^{-}\right)$, and Lemma 10 it follows that

$$
\int_{S^{n-1}} \frac{h(u)}{\kappa_{K}(u)} d S(u)=\int_{\operatorname{int} K_{o}}[h(-\nabla f(x), 1)+h(-\nabla g(x),-1)] d x .
$$

The desired result now follows from Lemma 11.

If in addition, $K$ is also origin-symmetric, then $g(-x)=f(x)$ for all $x \in \operatorname{int} K_{o}$. Now (15) shows that in this case Lemma 12 becomes:

Lemma 13. Suppose $K \subset \mathbb{R}^{n-1} \times \mathbb{R}$ is a smooth origin-symmetric convex body given by

$$
K=\left\{(x, t) \in \mathbb{R}^{n-1} \times \mathbb{R}:-g(x) \leq t \leq f(x), x \in K_{o}\right\},
$$

where $K_{o}$ is the image of the orthogonal projection of $K$ onto $\mathbb{R}^{n-1}$ and $f, g: K_{o} \rightarrow \mathbb{R}$. Then the support function of $\Pi_{p} K$ at $(y, t) \in \mathbb{R}^{n-1} \times \mathbb{R}$ is given by

$$
h_{\Pi_{p} K}^{p}(y, t)=\frac{2}{n \omega_{n} c_{n-2, p}} \int_{\operatorname{int} K_{o}}|t-y \cdot \nabla f(x)|^{p}\langle f\rangle(x)^{1-p} d x
$$


and

$$
h_{\Pi_{p} K}^{p}(y, t)=\frac{2}{n \omega_{n} c_{n-2, p}} \int_{\operatorname{int} K_{o}}|t+y \cdot \nabla g(x)|^{p}\langle g\rangle(x)^{1-p} d x .
$$

Lemma 14. Suppose $1<p<\infty$ and $K$ is a smooth originsymmetric convex body in $\mathbb{R}^{n}$. If $\xi$ is a subspace of codimension 1 , then

$$
\mathrm{S}_{\xi} \Pi_{p}^{*} K \subset \Pi_{p}^{*} \mathrm{~S}_{\xi} K
$$

with equality if and only if the chords of $K$ orthogonal to $\xi$ have midpoints that are coplanar.

Proof. Without loss of generality assume $\xi=\mathbb{R}^{n-1}$ and that $K \subset$ $\mathbb{R}^{n-1} \times \mathbb{R}$ is given by

$$
K=\left\{(x, t) \in \mathbb{R}^{n-1} \times \mathbb{R}:-g(x) \leq t \leq f(x), x \in K_{o}\right\},
$$

where $K_{o}$ is the image of the orthogonal projection of $K$ onto $\mathbb{R}^{n-1}$ and $f, g: K_{o} \rightarrow \mathbb{R}$, while

$$
\begin{aligned}
\mathrm{S}_{\mathbb{R}^{n-1}} K & = \\
\{(x, t) & \left.\in \mathbb{R}^{n-1} \times \mathbb{R}:-\frac{1}{2}(f(x)+g(x)) \leq t \leq \frac{1}{2}(f(x)+g(x)), x \in K_{o}\right\} .
\end{aligned}
$$

Now suppose

$$
h_{\Pi_{p} K}(y, t)=1=h_{\Pi_{p} K}(y,-s), \text { with } t \neq-s .
$$

Since $K$ is smooth and origin-symmetric, obviously so is $\mathrm{S}_{\mathbb{R}^{n-1}} K$. Now Lemma 13, the triangle inequality, Lemma 8, and Lemma 13 again, give

$$
\begin{aligned}
& h_{\Pi_{p} \mathrm{~S}_{\xi} K}^{p}\left(y, \frac{1}{2} t+\frac{1}{2} s\right) \\
& =\frac{2}{n \omega_{n} c_{n-2, p}} \int_{\operatorname{int} K_{o}}\left|\left(\frac{1}{2} t+\frac{1}{2} s\right)-y \cdot \nabla\left(\frac{1}{2} f+\frac{1}{2} g\right)(x)\right|^{p}\left\langle\frac{1}{2} f+\frac{1}{2} g\right\rangle(x)^{1-p} d x \\
& \leq \frac{1}{n \omega_{n} c_{n-2, p}} \int_{\operatorname{int} K_{o}}(|t-y \cdot \nabla f(x)|+|s-y \cdot \nabla g(x)|)^{p}(\langle f\rangle(x)+\langle g\rangle(x))^{1-p} d x \\
& \leq \frac{1}{n \omega_{n} c_{n-2, p}} \int_{\operatorname{int} K_{o}}\left[|t-y \cdot \nabla f(x)|^{p}\langle f\rangle(x)^{1-p}+|s-y \cdot \nabla g(x)|^{p}\langle g\rangle(x)^{1-p}\right] d x \\
& =\frac{1}{2} h_{\Pi_{p} K}^{p}(y, t)+\frac{1}{2} h_{\Pi_{p} K}^{p}(y,-s) \\
& =1 .
\end{aligned}
$$


Thus $h_{\Pi_{p} \mathrm{~S}_{\xi} K}\left(y, \frac{1}{2} t+\frac{1}{2} s\right) \leq 1$ which by Lemma 9 yields the desired inclusion.

If $\mathrm{S}_{\xi} \Pi_{p}^{*} K=\Pi_{p}^{*} \mathrm{~S}_{\xi} K$ then by Lemma 9 , we have

$$
h_{\Pi_{p}^{*} \mathrm{~S}_{\xi} K}^{p}\left(y, \frac{1}{2} t+\frac{1}{2} s\right)=1
$$

which would force equality in the inequalities above. The equality conditions of Lemma 8 now forces

$$
|t-y \cdot \nabla f(x)|\langle g\rangle(x)=|s-y \cdot \nabla g(x)|\langle f\rangle(x)
$$

for all $x \in \operatorname{int} K_{o}$. Choose $y=0$ and (since $s, t$ are such that $(0, t),(0,-s)$ $\left.\in \partial \Pi_{p}^{*} K\right)$ we must have $s=t$ and thus $\langle g\rangle(x)=\langle f\rangle(x)$ for all $x \in \operatorname{int} K_{o}$. But

$$
\langle f-g\rangle=0
$$

implies that $f-g$ is linear and hence that the chords of $K$ orthogonal to $\mathbb{R}^{n-1}$ have coplanar midpoints.

The fact that the coplanarity of the midpoints of the chords of $K$ that are orthogonal to $\xi$ forces $\mathrm{S}_{\xi} \Pi_{p}^{*} K=\Pi_{p}^{*} \mathrm{~S}_{\xi} K$ is left to the reader (and will not be used in this article). q.e.d.

Note that an obvious limit argument shows the inclusion of Lemma 14 for $p>1$ and $K$ origin-symmetric and smooth immediately yields exactly the same inclusion for all $p \geq 1$ and all arbitrary origin-symmetric bodies $K$. However, the critical equality conditions are lost in the limit process.

\section{Proofs of the theorems}

Since the volume of convex bodies is obviously unaffected by Steiner symmetrization, Lemma 14 and the definition of $\mathrm{p}_{p}$ immediately yield:

Lemma 15. If $K$ is a smooth origin-symmetric convex body and $\xi$ is a codimension 1 subspace, then

$$
\mathrm{p}_{p}(K) \leq \mathrm{p}_{p}\left(\mathrm{~S}_{\xi} K\right)
$$

with equality if and only if the chords of $K$ orthogonal to $\xi$ have coplanar midpoints.

Now a body at which $\mathrm{p}_{p}$ attains a maximum must be (by our class reduction arguments) both origin-symmetric and smooth. But 
the body's maximality together with Lemma 15 shows that any parallel set of chords of the body must have coplanar midpoints. The classical Bertrand-Brunn theorem now allows us to conclude that this maximal body can only be an ellipsoid. This proves Theorem 2 .

Lemma 5 shows that Theorem 2 immediately gives Theorem 1.

\section{Open problems}

Question. Suppose $1 \leq p<\infty$ and $K$ is a convex body in $\mathbb{R}^{n}$. If $\xi$ is a subspace of $\mathbb{R}^{n}$ of codimension 1 , then is it the case that

$$
\mathrm{S}_{\xi} \Pi_{p}^{*} K \subset \Pi_{p}^{*} \mathrm{~S}_{\xi} K
$$

with equality if and only if the chords of $K$ orthogonal to $\xi$ have midpoints that are coplanar?

A positive answer to this question would immediately and directly yield a proof of Theorem 2 (and thus Theorem 1 as well).

Conjecture. If $K$ is a convex body such that $\Gamma_{p} \Pi_{p}^{*} K$ is a dilate of $K$, then $K$ must be an ellipsoid.

A proof of this together with Lemma 7 would immediately prove Theorem 2 (and thus Theorem 1 as well). Obviously, the conjecture is true for $p=2$.

\section{References}

[1] W. Blaschke, Affine geometrie XIV, Ber. Verh. Sächs. Akad. Wiss. Leipzig Math.-Phys. Kl 70 (1918) 72-75.

[2] E. D. Bolker, A class of convex bodies, Trans. Amer. Math. Soc. 145 (1969) 323-345.

[3] J. Bourgain \& J. Lindenstrauss, Projection bodies, Geometric Aspects of Functional Analysis (J. Lindenstrauss and V. D. Milman, eds.) Springer Lecture Notes in Math. 1317 (1988) 250-270.

[4] J. Bourgain \& V. Milman, New volume ratio properties for convex symmetric bodies in $R^{n}$, Invent. Math. 88 (1987) 319-340.

[5] H. Busemann, Volume in terms of concurrent cross-sections, Pacific J. Math. 3 (1953) 1-12.

[6] Wm. J. Firey, p-means of convex bodies, Math. Scand. 10 (1962) 17-24. 
[7] R. J. Gardner, A positive answer to the Busemann-Petty problem in three dimensions, Ann. of Math. 140 (1994) 435-447.

[8] _ Geometric tomography, Cambridge Univ. Press, Cambridge, 1995.

[9] P. R. Goodey \& W. Weil, Centrally symmetric convex bodies and the spherical Radon transform, J. Differential Geom. 35 (1992) 675-688.

[10] Z Z Z _ _ ber and J. M. Wills, eds.) North-Holland, Amsterdam, 1993, 1297-1326.

[11] A. Koldobsky, Generalized Lévy representation of norms and isometric embeddings into $L_{p}$-spaces, Ann. Inst. H. Poincaré Probab. Statist. 28 (1992) 335-353.

[12] Common subspaces of $L_{p}$-spaces, Proc. Amer. Math. Soc. 122 (1994) $207-212$.

[13] _ Positive definite functions, stable measures, and isometries on Banach spaces, Dekker Lecture Notes in Pure Appl. Math 175 (1996) 275-290.

[14] _ A Banach subspace of $L_{1 / 2}$ which does not embed in $L_{1}$ (isometric version), Proc. Amer. Math. Soc. 124 (1996) 155-160.

[15] - Inverse formula for the Blaschke-Levy representation, Houston J. Math. 23 (1997) 95-108.

[16] The Schoenberg problem on positive-definite functions, St. Petersburg Math. J. 3 (1992) 563-570.

[17] K. Leichtweiß, Affine geometry of convex bodies, J. A. Barth, Heidelberg, 1998.

[18] J. Lindenstrauss \& V. D. Milman, Local theory of normed spaces and convexity, Handbook of Convex Geometry, (P. M. Gruber and J. M. Wills eds.), NorthHolland, Amsterdam, 1993. 1149-1220.

[19] E. Lutwak, On some affine isoperimetric inequalities, J. Differential Geom. 23 (1986) 1-13.

[20] Centroid bodies and dual mixed volumes, Proc. London Math. Soc. 60 (1990) 365-391.

[21] _ The Brunn-Minkowski-Firey theory I: mixed volumes and the Minkowski problem, J. Differential Geom. 38 (1993) 131-150.

[22] _ The Brunn-Minkowski-Firey theory II: Affine and geominimal surface areas, Adv. in Math. 118 (1996) 244-294.

[23] E. Lutwak, D. Yang \& G. Zhang, A new ellipsoid associated with convex bodies, Duke Math. J. 104 (2000) 375-390.

[24] E. Lutwak \& G. Zhang, Blaschke-Santaló inequalities, J. Differential Geom. 47 (1997) 1-16. 
[25] V. D. Milman \& A. Pajor, Cas limites des inégalités du type Khinchine et applications géométriques, C.R. Acad. Sci. Paris 308 (1989) 91-96.

[26] _ Isotropic position and inertia ellipsoids and zonoids of the unit ball of a normed n-dimensional space, Geometric Aspects of Functional Analysis (J. Lindenstrauss and V.D. Milman, eds.) Springer Lecture Notes in Math. 1376 (1989) 64-104.

[27] C. M. Petty, Centroid surfaces, Pacific J. Math. 11 (1961) 1535-1547.

[28] , Projection bodies, Proc. Coll. Convexity, Copenhagen, 1965, Københavns Univ. Mat. Inst., 1967, 234-241.

[29] - Isoperimetric problems, Proc. Conf. Convexity and Combinatorial Geometry (Univ. Oklahoma, 1971), University of Oklahoma, 1972, 26-41.

[30] B. Rubin, Inversion of fractional integrals related to the spherical Radon transform, J. Funct. Anal. 157 (1998) 470-487.

[31] R. Schneider, Zu einem Problem von Shephard über die Projectionen konvexer Körper, Math. Z. 101 (1967) 71-82.

[32] Convex bodies: the Brunn-Minkowski theory, Cambridge Univ. Press, Cambridge, 1993.

[33] R. Schneider \& W. Weil, Zonoids and related topics, Convexity and its applications, (P. M. Gruber and J. M. Wills, eds.), Birkhäuser, Basel, 1983, 296-317.

[34] A. C. Thompson, Minkowski Geometry, Cambridge Univ. Press, Cambridge, 1996.

[35] G. Zhang, Restricted chord projection and affine inequalities, Geom. Dedicata 39 (1991) 213-222.

[36] _ Centered bodies and dual mixed volumes, Trans. Amer. Math. Soc. 345 (1994) 777-801.

[37] Intersection bodies and the Busemann-Petty inequalities in $\mathbb{R}^{4}$, Ann. of Math. 140 (1994) 331-346.

[38] A positive solution to the Busemann-Petty problem in $\mathbb{R}^{4}$, Ann. of Math. 149 (1999) 535-543.

Polytechnic University, Brooklyn, NY 